\title{
MicroRNA-34a induces apoptosis in PC12 cells by reducing B-cell lymphoma 2 and sirtuin-1 expression
}

\author{
QIANG LIN ${ }^{1,2}$, YURONG MAO ${ }^{1,2}$, YUNLIN SONG $^{3}$ and DONGFENG HUANG ${ }^{1,2}$ \\ ${ }^{1}$ Department of Rehabilitation Medicine; ${ }^{2}$ Guangdong Engineering Technology Research Center \\ for Rehabilitation Medicine and Clinical Translation, The First Affiliated Hospital, Sun Yat-Sen University, \\ Guangzhou, Guangdong 510080; ${ }^{3}$ Intensive Care Unit, The First Affiliated Hospital of Xinjiang Medical University, \\ Ürümqi, Xinjiang Uyghur Autonomous Region 830011, P.R. China
}

Received September 6, 2014; Accepted July 3, 2015

DOI: $10.3892 / \mathrm{mmr} .2015 .4185$

\begin{abstract}
MicroRNA-34a (miR-34a) is a direct target of p53 and was reported to induce cell cycle arrest, apoptosis and senescence. Inhibition of the NAD-dependent deacetylase sirtuin-1 (SIRT1) by miR-34a leads to an increase in acetylated p53, which promotes cell apoptosis. B-cell lymphoma 2 (Bcl-2) is also involved in apoptosis, and was originally characterized with respect to its role in controlling outer mitochondrial membrane integrity. The effect of miR-34a in PC12 cells has not yet been reported. In the present study, it was hypothesized that Bcl-2 and SIRT1 may be critical downstream targets of miR-34a that participate in apoptosis induction. miR-34a mimics and inhibitors were transfected into PC12 cells, and the apoptosis and proliferation rates were compared between groups. It was demonstrated that induction of miR-34a promotes apoptosis and senescence, inhibits proliferation, and leads to marked alterations in SIRT1, Bcl-12 and acetyl (ac)-p53 expression. These data indicate that miR-34a may be important in neuropathy.
\end{abstract}

\section{Introduction}

MicroRNAs (miRs) are small, 19-25 nucleotide long non-coding RNAs that function as critical regulators of gene expression. They bind to the 3'-untranslated region (UTR) of target genes and, along with other accessory proteins, form an RNA-induced silencing complex that is responsible for promoting mRNA degradation and inhibiting mRNA translation (1). Increasing evidence indicates that miRNAs are essential in a number of biological processes, including development, apoptosis, cell proliferation, differentiation, disease

Correspondence to: Professor Dongfeng Huang, Department of Rehabilitation Medicine, The First Affiliated Hospital, Sun Yat-Sen University, 58 Zhongshan Road, Guangzhou, Guangdong 510080, P.R. China

E-mail: huangdf@mail.sysu.edu.cn

Key words: microRNA-34a, PC12 cells, B-cell lymphoma 2, NAD-dependent deacetylase, sirtuin-1, apoptosis survival and cell death (2-4). Aberrantly expressed miRNAs are also associated with several neurological diseases, such as Parkinson's disease, dementia and glioma (5-8). Current research has revealed that ectopic overexpression of microRNA-34a (miR-34a) can induce cell cycle arrest, apoptosis and senescence to inhibit cancer recurrence, migration and metastasis $(9,10)$. Multiple studies have also indicated that miR-34a regulates a variety of target mRNAs, including cyclin-dependent kinase 4/6 (CDK4/6), E2F transcription factor3 (E2F3), Cyclin E2, B-cell lymphoma 2 (Bcl-2) and NAD-dependent deacetylase sirtuin-1 (SIRT1) $(11,12)$. TP53 is one of the most common apoptosis-related genes in mammalian cells, and its gene product p53, activates the transcription of a set of miRNAs, including members of the miR-34 family (13). Bcl-2 is another apoptosis-related gene. Bcl-2 family members were originally characterized with respect to their roles in regulating apoptosis through complex interactions that dictate the integrity of the outer mitochondrial membrane. As an NAD-induced deacetylase, SIRT1 is a transcriptional regulator and can inhibit the expression of pro-apoptotic proteins (14). SIRT1 regulates p53-dependent apoptosis by deacetylating and destabilizing p53. It has been confirmed that SIRT1 mediates miR-34a-induced apoptosis by regulating p53 activity. A positive feedback loop has been identified, in which p53 induces expression of miR-34a, suppressing SIRT1 and increasing p53 activity (15). PC12 cells are derived from rat adrenal medulla pheochromocytoma, and are widely utilized in in vitro studies of neurological diseases. However, thus far, there have been no experimental studies of the effect of miR-34a in PC12 cells.

It was hypothesized that Bcl-2 and SIRT1 may be critical downstream targets of miR-34a that participate in cellular apoptosis. In the present study, miR-34a mimics or inhibitors were transfected into PC12 cells, and the apoptosis and proliferation rates were measured. The aim of the present study was to establish whether miR-34a-induced PC12 cell apoptosis occurs via suppression of SIRT1 and Bcl-2.

\section{Materials and methods}

Cell culture. PC12 cells (obtained from the Biomedical Laboratory of Xinjiang Medical University, Ürümqi, China) 
were cultured in RPMI medium (GE Healthcare, Logan, UT, USA) containing 10\% horse serum (Hangzhou Sijiqing Biological Engineering Materials Co., Hangzhou, China) and 5\% fetal bovine serum (Hangzhou Sijiqing Biological Engineering Materials Co.,) in a $\mathrm{CO}_{2}$ humidified incubator at $37^{\circ} \mathrm{C}$. Transfection was performed using Lipofectamine 2000 (Invitrogen Life Technologies, Carlsbad, CA, USA) kit according to the manufacturer's instructions. The cells were divided into the following groups: Negative control group (control group), $100 \mathrm{nM}$ miR-34a mimic (miR-34a mimic group) and $100 \mathrm{nM}$ miR-34a inhibitor (miR-34a inhibitor group). The miR-34a mimic and inhibitor were obtained from Shanghai Genechem Co., Ltd. (Shanghai, China).

3-(4,5-dimethylthiazol-2-yl)-2-5 diphenyltetrazolium bromide (MTT) assay. PC12 cells were seeded into 96-well plates at a density of $4 \times 10^{3}$ cells/well. The effect of miR-34a on cell growth and viability was determined by an MTT assay. After transfection $(24,48$ or $72 \mathrm{~h})$ with either miR-34a mimic or miR-34a inhibitor, cells were incubated with MTT $(5 \mathrm{mg} / \mathrm{ml})$ in phosphate-buffered saline (PBS) for $4 \mathrm{~h}$, and then lysed with $50 \% \mathrm{~N}, \mathrm{~N}$ dimethylformamide and 10\% SDS for an additional $3 \mathrm{~h}$ at $37^{\circ} \mathrm{C}$. The absorbance was measured at $570 \mathrm{~nm}$ using an ELISA reader (DG-3022; Nanjing Huangdong Electronic Information \& Technology Co., Ltd, Nanjing, China). Samples were plated in triplicate, and the average value for each group was calculated.

Senescence-associated $\beta$-galactosidase staining. After transfection with miR-34a mimics or inhibitors, PC12 cells were stained for SA- $\beta$-gal activity analysis. Cells were fixed with $4 \%$ formaldehyde for $15 \mathrm{~min}$ at room temperature, washed three times with PBS, and incubated with $1 \mathrm{ml} \mathrm{X}$-gal solution (Hangzhou Sijiqing Biological Engineering Materials Co.) for $12 \mathrm{~h}$ at $37^{\circ} \mathrm{C}$, avoiding exposure to $\mathrm{CO}_{2}$. Following incubation, a blue color developed in senescent cells, observed under a microscope (IX71; Olympus Corporation, Tokyo, Japan) and the proportions of senescent cells were observed by digital imaging (Motic Images Plus 2.0; Motic China Group Co., Ltd., Xiamen, China).

Apoptosis analysis by fluorescent-activated cell sorting. After transfection with miR-34a mimics or inhibitors, PC12 cells were harvested, washed with ice-cold PBS, resuspended in $500 \mu \mathrm{l}$ binding buffer, and incubated with $5 \mu \mathrm{l}$ propidium iodide (PI; Beyotime Institute of Biotechnology, Jiangsu, China) and $5 \mu$ l Annexin V-fluorescein isothiocyanate (FITC; Beyotime Institute of Biotechnology) for $10 \mathrm{~min}$ in the dark. The cells were then washed and resuspended in $500 \mu \mathrm{l}$ PBS, and cell apoptosis was analyzed by flow cytometry (using an Epics XL-MCL flow cytometer; Beckman Coulter, Inc., Brea, CA,USA).

Western blot analysis. PC12 cell lysates were extracted using a BCA Protein Assay Reagent kit (Beyotime Institute of Biotechnology, Shanghai, China), following mimic or inhibitor treatment. Protein concentration was determined using the bicinchoninic acid protein assay reagent (Beyotime Institute of Biotechnology). Equal quantities of protein from each sample were loaded and electrophoresed on $10 \%$ SDS-PAGE

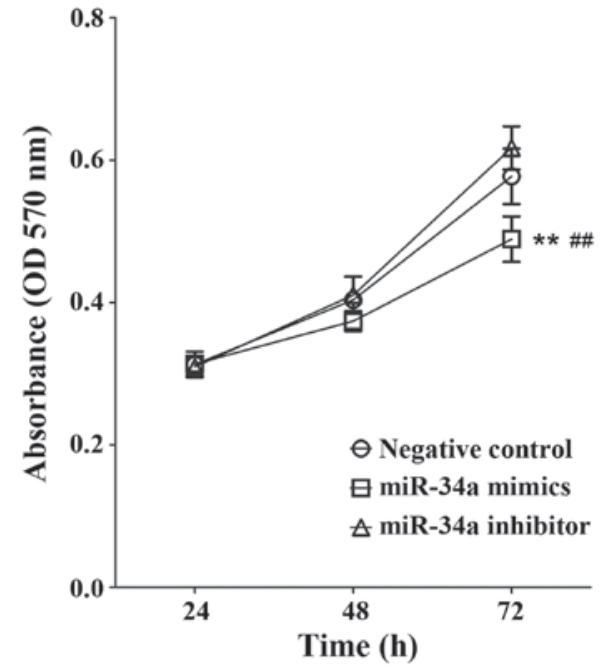

Figure 1. Effects of miR-34a mimics and inhibitors on cell proliferation determined by 3-(4,5-dimethylthiazol-2-yl)-2-5 diphenyltetrazolium bromide assays. Data are expressed as the mean \pm standard deviation, ${ }^{* *} \mathrm{P}<0.01$, compared with the negative control group and ${ }^{\# \#} \mathrm{P}<0.01$ compared with the miR-34a inhibitor group, $n=5$ for each group. miR, microRNA; OD, optical density.

gels (Beyotime Institute of Biotechnology), and transferred to nitrocellulose membranes [Sangon Biotech (Shanghai) Co., Ltd., Shanghai, China]. After blocking with non-fat dried milk, membranes were probed with polyclonal rabbit anti-human acetyl-p53 [dilution, 1:1,000 (cat. no. CS2525); Cell Signaling Technology, Inc., Danvers, MA, USA], polyclonal rabbit anti-human Bcl-2 [dilution, 1:1,000 (cat. no. BS4023); Bioworld Technology, Inc., St. Louis Park, MN, USA] or polyclonal rabbit anti-human SIRT1 [dilution, 1:1,000 (cat. no. CS2327); Cell Signaling Technology, Inc.] antibodies. Antibody signals were visualized using a Chemiluminescent Detection kit, according to the manufacturer's instructions (Beyotime Institute of Biotechnology). Experiments with blank and negative controls were conducted in parallel. The relative band intensities of the blots were quantified with Adobe Photoshop software (Adobe Systems, Inc., San Jose, CA, USA)..

Statistical analysis. All experiments were repeated at least three times. All values are expressed as the mean \pm standard deviation. The difference between means was analyzed by Student's unpaired t-test. $\mathrm{P}<0.05$ was considered to indicate a statistically significant difference. All statistical analyses were conducted using SPSS 19.0 (SPSS, Inc., Armonk, NY, USA).

\section{Results}

miR-34a mimics inhibit the proliferation of PC12 cells. To assess the biological role of miR-34a during the proliferation of PC12 cells, cells were transiently transfected with miR-34a mimics or inhibitors, and the proliferation was measured 24, 48 and $72 \mathrm{~h}$ following transfection using an MTT assay. As shown in Fig. 1, following transfection with miR-34a mimics, the proliferation of PC12 cells was significantly decreased compared with that of the negative control group $(\mathrm{P}<0.01)$. The proliferation of the miR-34a inhibitor group was marginally 
A :

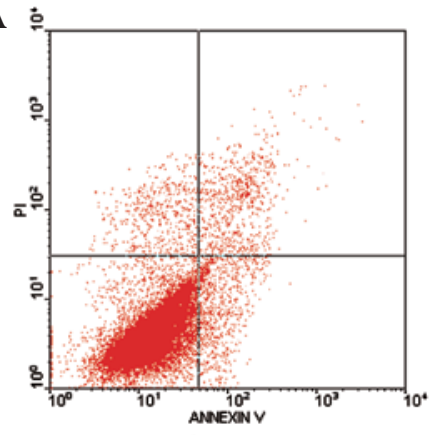

Negative control

C

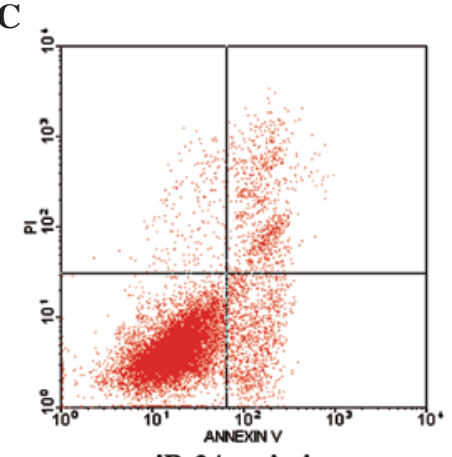

miR-34a mimics
B

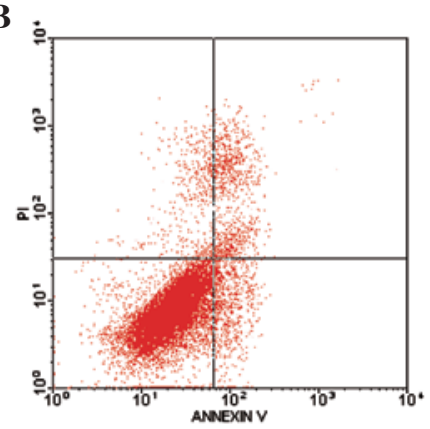

miR-34a inhibitor

D

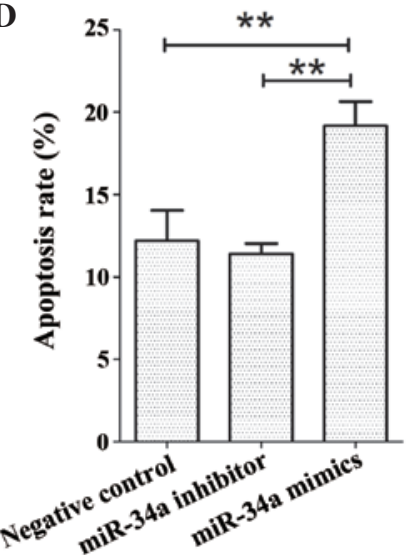

Figure 2. Effects of miR-34a mimics and inhibitors on cell apoptosis rates analyzed by fluorescence-activated cell sorting. Cells were dually stained with Annexin V/PI and analyzed by flow cytometry to determine the population of cells in early and late apoptosis stages in the (A) negative control, (B) cells treated with miR-34a inhibitor and (C) cells treated with miR-34a mimics. Left lower quadrant, viable cells; right lower quadrant, early apoptotic cells; right upper quadrant, late apoptotic cells; left upper quadrant, necrotic cells. (D) Quantification of the total percentage of apoptotic cells in each group. * $\mathrm{P}<0.01, \mathrm{n}=3$ for each group. miR, microRNA; PI, propidium iodide.
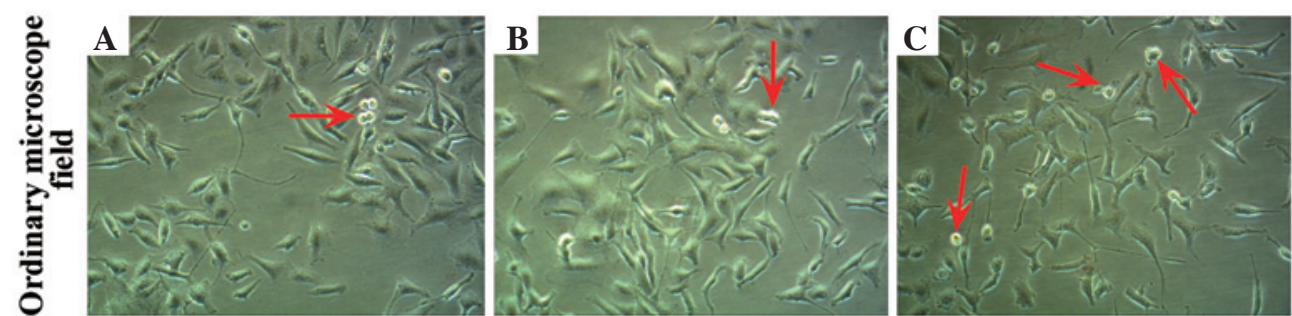

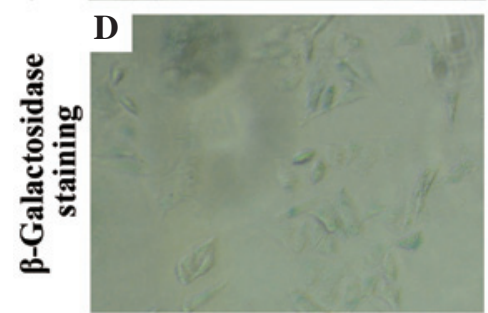

miR-34a inhibitor

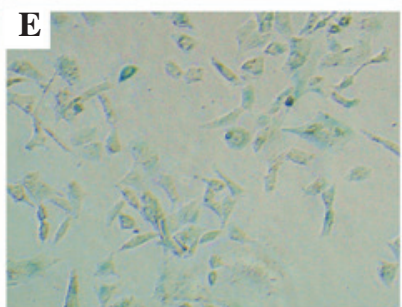

Negative control

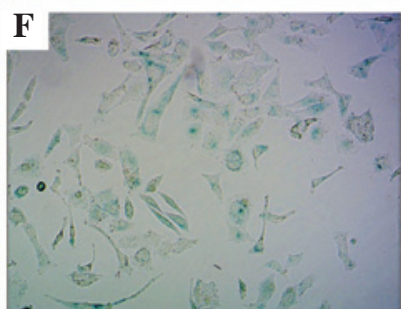

miR-34a mimics

Figure 3. Morphology of PC12 cells (A) after transfection with an miR-34a inhibitor (B) in the negative control group or (C) following transfection with miR-34a mimic. (A-C) The arrows indicate the non-adherent cells, which may be the apoptotic or senescent cells (magnifications, $\mathrm{x} 40$ ). Representative images of SA $\beta$-gal staining of PC12 cells in (D) the miR-34a inhibitor group, (E) negative control group and (F) the miR-34a mimic group. Blue staining indicates the SA- $\beta$-gal-positive cells.

higher than that of the negative control group; however, no significant difference was identified $(\mathrm{P}>0.05)$.

miR-34a induces PC12 cell apoptosis. It is well known that miR-34a is an important component of the p53 tumor suppressor protein transcriptional network, which regulates cell proliferation and cell cycle progression. In order to investigate the biological effects of miR-34a in nerve cells, PC12 cells were transiently transfected with miR-34a mimics or miR-34a inhibitors, and the proportions of apoptotic cells were quantified using 

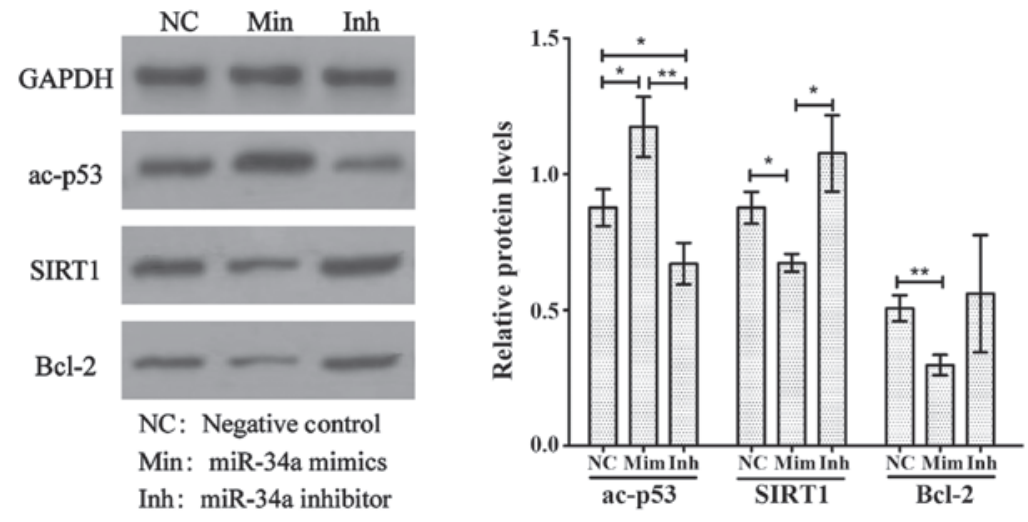

Figure 4. Protein expression levels of SIRT1, Bcl-2 and ac-p53. The protein levels were determined by western blot analysis, using GAPDH levels as a loading control. All western blot data are presented as the mean \pm standard deviation $(\mathrm{n}=3),{ }^{*} \mathrm{P}<0.05$ and ${ }^{* *} \mathrm{P}<0.01$. SIRT1, sirtuin 1 ; Bcl-2, B-cell lymphoma 2; miR, microRNA.

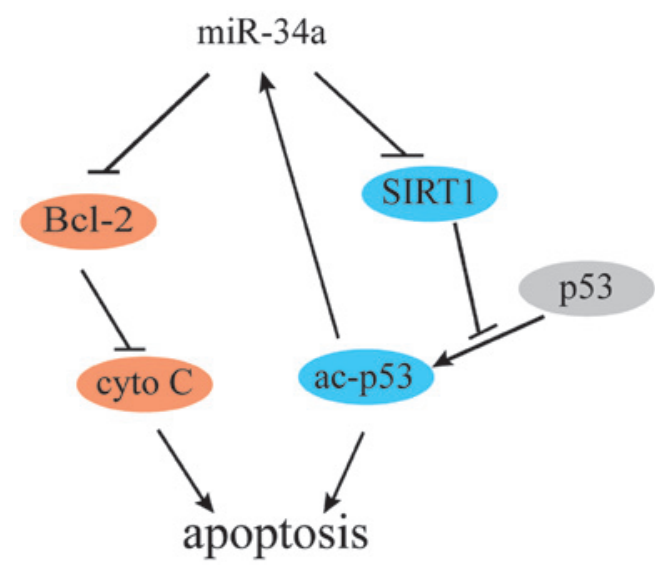

Figure 5. Proposed feedback loop and apoptosis mechanism involving miR-34a, p53, SIRT1 and Bcl-2. miR-34a, microRNA-34a; SIRT1, sirtuin 1; Bcl-2, B-cell lymphoma 2; cyto C, cytochrome $c$.

an Annexin V-FITC/PI dual staining assay. As shown in Fig. 2, after transfection, the total proportion of apoptotic cells in the miR-34a mimic group was significantly increased compared with that of the control group $(\mathrm{P}<0.01)$. The apoptotic rate of miR-34a inhibitor group was marginally lower than that of the control group, however, no significant difference was identified $(\mathrm{P}>0.05)$. The total apoptotic rates for control, miR-34a inhibitor and miR-34a mimic transfection groups were $12.2 \pm 1.06$, $11.4 \pm 0.34$ and $19.2 \pm 0.84 \%$, respectively.

miR-34a induces PC12 cell senescence. The influence of miR-34a on cell senescence was then evaluated using the $\beta$-galactosidase staining assay. The number of non-adherent cells in the miR-34a mimic group was increasing compared with the miR-34a inhibitor group and the control group, however, no significant difference was identified between the miR-34a inhibitor group and the control group (Fig. 3A-C). SA- $\beta$-gal staining analysis showed that the miR-34a mimics greatly increased SA- $\beta$-gal activity (Fig. 3D-F). These results demonstrate that miR-34a increases PC12 cell apoptosis and senescence.

miR-34a reduces the expression of Bcl-2 and SIRT1. To determine whether miR-34a expression levels correlate with the ac-p53, Bcl-2 and SIRT1 levels in PC12 cells, the expression of each protein was quantified by western blot analysis after transfection with miR-34a mimics or inhibitors. As Fig. 4 shows, compared with the control group, the expression levels of Bcl-2 $(\mathrm{P}<0.01)$ and SIRT1 $(\mathrm{P}<0.05)$ in the miR-34a mimic group were significantly reduced, while the levels of ac-p53 $(\mathrm{P}<0.05)$ were elevated in this group. In addition, the ac-p53 in miR-34a inhibitor group was reduced compared with the control group $(\mathrm{P}<0.05)$. The levels of SIRT1 in the miR-34a inhibitor group were significantly elevated compared with the miR-34a mimics group $(\mathrm{P}<0.05)$, however, the SIRT1 levels were not significantly different from the control group. The levels of Bcl-2 in the miR-34a inhibitor group were marginally elevated compared with the miR-34a mimic and the control groups, however the difference was not significant.

\section{Discussion}

$\mathrm{PC} 12$ is a cell line derived from a pheochromocytoma of the rat adrenal medulla, which contains a mixture of neuroblastic and eosinophilic cells (16). PC12 cells stop dividing and terminally differentiate when treated with nerve growth factor or dexamethasone, rendering PC12 cells a good in vitro model for investigating neuronal differentiation and neurosecretion $(17,18)$. Additionally, due to their widespread availability and transfectable features, $\mathrm{PC} 12$ cells are one of the most commonly used models for investigating the physiology, pathology and pharmacology of neural cell differentiation. In recent years, researchers have developed various neurodegenerative disease cell models, including Alzheimer's and Parkinson's disease models, using PC12 cells (19,20). As small, endogenously expressed non-coding RNAs, miRNAs regulate gene expression by promoting the degradation of target mRNA and inhibiting translation. miRNAs are frequently involved in the regulation of cellular differentiation, proliferation, metabolism and apoptosis. As a member of the miR-34 family, miR-34a has been widely investigated in recent years. Several studies have indicated that upregulation of miR-34a expression can induce apoptosis, senescence, differentiation, cell cycle arrest and growth suppression $(21,22)$. Overexpression of miR-34a increases the proportion of postmitotic neurons of mouse neural stem cells (23). SIRT1 is a nicotinamide 
adenine dinucleotide (NAD)-dependent histone deacetylase that has been implicated in inflammation, circadian rhythms, hypoxic responses, cell survival, life longevity and metabolic processes $(24,25)$. SIRT1 also exhibits a protective role in certain neurodegenerative disease models (26). It has been reported that SIRT1 inhibits lipopolysaccharide-mediated proinflammatory cytokine release in microglia and circumvents dopaminergic neuronal injury induced by activated microglial-derived factors via p53-caspase-3-dependent apoptosis, which indicates that upregulation of SIRT1 may provide a promising target for therapeutic intervention in neuroinflammatory diseases (27).

p53 is a sensor of chronic and acute alterations in cellular physiology and interacts with DNA to aid in regulating chromosomal integrity (28). miR-34a enhances p53 activity by reducing p53 deacetylation, which in turn results in a decrease in SIRT1 expression (15). This decrease is achieved at the post-transcriptional level, involving miRNA binding to the 3'-UTR of SIRT1. In addition, the inhibition of SIRT1 activates p53-dependent apoptosis through deacetylation and stabilization of p53. As an important anti-apoptosis gene, Bcl-2 cooperates with apoptosis activating factors and forms the Bcl-2-Apaf-1-caspase 9 complex, which inhibits the activation of caspases 9 and 3 , and prevents the initiation of mitochondrial apoptosis access. A study showed that miR-34a inhibits the function and activity of Bcl-2 and promotes cellular apoptosis (29).

As demonstrated in the present study, transfection of PC12 cells with miR-34a mimics resulted in insignificant alterations in cell viability compared with the control group. Apoptosis analysis by flow cytometry revealed that the apoptosis rate of the miR-34a mimic group was significantly higher compared with the control group. Consistently, compared with the control group, the expression of Bcl-2 and SIRT1 in the miR-34a mimic group was decreased, while the ac-p53 was increased. Cell senescence is a form of stagnation of cell growth and, the results obtained using the SA- $\beta$-gal staining assay showed that the miR-34a mimic greatly increased the SA- $\beta$-gal activity, suggesting that the miR34a mimic could increase the senescence of PC12 cells. miR-34a binds to the 3'-UTR sequences of SIRT1 mRNA, immediately inhibiting the translation of SIRT1. The decrease in SIRT1 expression leads to an increase in the acetylation of p53, enhancing p53 transcriptional activity, through the positive feedback loop as shown in Fig. 5. As a highly-conserved $\mathrm{NAD}^{+}$induced deacetylase enzyme, SIRT1 was first identified in yeast, where, through regulation of downstream targets, such as p53, Foxo and Ku79, it is involved in reducing oxidative stress and apoptosis, and regulating gene silencing and cell cycle progression (30-32). SIRT1 promotes the deacetylation of c-terminal lysine 382 of the p53 protein, regulating the transcriptional activity of p53 and inhibiting p53-induced apoptosis (33). It is also reported that p53 can inhibit autophagy by reducing expression of SestrinZ and Draln, and accelerating cellular senescence $(34,35)$. Studies have revealed that the deposition of $\beta$-amyloid protein $(A \beta)$ is a critical initiating factor in Alzheimer's disease, and $A \beta$ is a direct contributor to the neurofibrillary tangles in the brain and results in the loss of neurons (36). In models of Alzheimer's disease, high expression of SIRT1 in rat brains was found to strengthen the catabolic pathway of the amyloid protein, and reduce $A \beta$ deposition (37). The present study showed that transfection of miR-34a mimics can effectively reduce the expression of SIRT1 in PC12 cells, while transfection of miR-34a inhibitors can increase the expression of SIRT1. Therefore, it was demonstrated that miR-34a activates SIRT1, which, due to the roles of SIRT1 in Alzheimer's disease, indicates that miR-34a may aid in preventing and curing Alzheimer's disease.

In conclusion, the present data indicates that miR-34a induces PC12 cellular apoptosis, which may be associated with the inhibition of SIRT1 and Bcl-2. Furthermore, this study highlights the importance of the positive feedback loop formed by miR-34a-SIRT1-p53 in cellular apoptosis. These results reveal that miR-34a is a key regulator of cellular apoptosis and a potential therapeutic target in neuronal diseases.

\section{Acknowledgements}

This study has been supported by the National Science Foundation of China (grant no. 81372108).

\section{References}

1. Benfey PN: Molecular biology: Microrna is here to stay. Nature 425: 244-245, 2003.

2. Chen LH, Tsai KL, Chen YW, Yu CC, Chang KW, Chiou SH, $\mathrm{Ku} \mathrm{HH}$, Chu PY, Tseng LM and Huang PI: Microrna as a novel modulator in head and neck squamous carcinoma. J Oncol 2010: $135632,2010$.

3. Ozata DM, Caramuta S, Velazquez-Fernandez D, Akcakaya P, Xie H, Hoog A, Zedenius J, Backdahl M, Larsson C and Lui WO: The role of microrna deregulation in the pathogenesis of adrenocortical carcinoma. Endocr Relat Cancer 18: 643-655, 2011.

4. Guo Z, Chi F, Song Y, Wang C, Yu R, Wei T, Gui J and Zhu X: Transcriptome analysis of murine thymic epithelial cells reveals ageassociated changes in microrna expression. Int J Mol Med 32: 835-842, 2013.

5. Santosh PS, Arora N, Sarma P, Pal-Bhadra M and Bhadra U: Interaction map and selection of microrna targets in parkinson's disease-related genes. J Biomed Biotechnol 363145, 2009.

6. Sheinerman KS and Umansky SR: Circulating cell-free microrna as biomarkers for screening, diagnosis and monitoring of neurodegenerative diseases and other neurologic pathologies. Front Cell Neurosci 7: 150, 2013.

7. Ai J, Sun LH, Che H, Zhang R, Zhang TZ, Wu WC, Su XL, Chen X, Yang G, Li K, et al: Microrna-195 protects against dementia induced by chronic brain hypoperfusion via its anti-amyloidogenic effect in rats. J Neurosci 33: 3989-4001, 2013.

8. Men D, Liang $\mathrm{Y}$ and Chen L: Decreased expression of microrna-200b is an independent unfavorable prognostic factor for glioma patients. Cancer Epidemiol 38: 152-156, 2014

9. Liu C, Zhou C, Gao F, Cai S, Zhang C, Zhao L, Zhao F, Cao F, Lin J, Yang Y, et al: Mir-34a in age and tissue related radio-sensitivity and serum mir-34a as a novel indicator of radiation injury. Int J Biol Sci 7: 221-233, 2011.

10. Boon RA, Iekushi K, Lechner S, Seeger T, Fischer A, Heydt S, Kaluza D, Treguer K, Carmona G, Bonauer A, et al: Microrna-34a regulates cardiac ageing and function. Nature 495: 107-110, 2013.

11. Li L, Yuan L, Luo J, Gao J, Guo J and Xie X: Mir-34a inhibits proliferation and migration of breast cancer through down-regulation of bcl-2 and sirt1. Clin Exp Med 13: 109-117, 2013.

12. Tomosugi M, Sowa Y, Yasuda S, Tanaka R, Te RH, Ikawa H, Koyama $\mathrm{M}$ and Sakai T: Retinoblastoma gene-independent g1 phase arrest by flavone, phosphatidylinositol 3-kinase inhibitor and histone deacetylase inhibitor. Cancer Sci 103: 2139-2143, 2012.

13. Bommer GT, Gerin I, Feng Y, Kaczorowski AJ, Kuick R, Love RE, Zhai Y, Giordano TJ, Qin ZS, Moore BB, et al: P53-mediated activation of mirna34 candidate tumor-suppressor genes. Curr Biol 17: 1298-1307, 2007. 
14. Matsushita N, Takami Y, Kimura M, Tachiiri S, Ishiai M, Nakayama T and Takata M: Role of nad-dependent deacetylases sirtl and sirt 2 in radiation and cisplatin-induced cell death in vertebrate cells. Genes Cells 10: 321-332, 2005.

15. Castro RE, Ferreira DM, Afonso MB, Borralho PM, Machado MV, Cortez-Pinto $\mathrm{H}$ and Rodrigues CM: Mir-34a/sirt1/p53 is suppressed by ursodeoxycholic acid in the rat liver and activated by disease severity in human non-alcoholic fatty liver disease. J Hepatol 58: 119-125, 2013.

16. Attiah DG, Kopher RA and Desai TA: Characterization of pc12 cell proliferation and differentiation-stimulated by ecm adhesion proteins and neurotrophic factors. J Mater Sci Mater Med 14: 1005-1009, 2003.

17. Leoni C, Menegon A, Benfenati F, Toniolo D, Pennuto M and Valtorta F: Neurite extension occurs in the absence of regulated exocytosis in pc12 subclones. Mol Biol Cell 10: 2919-2931, 1999.

18. Taupenot L: Analysis of regulated secretion using pc12 cells. Curr Protoc Cell Biol 15: 12-15, 2007.

19. Martin D, Salinas M, Lopez-Valdaliso R, Serrano E, Recuero M and Cuadrado A: Effect of the alzheimer amyloid fragment abeta (25-35) on akt/pkb kinase and survival of pc12 cells. J Neurochem 78: 1000-1008, 2001.

20. Zhang ZT, Cao XB, Xiong N, Wang HC, Huang JS, Sun SG and Wang T: Morin exerts neuroprotective actions in parkinson disease models in vitro and in vivo. Acta Pharmacol Sin 31: 900-906, 2010.

21. Hermeking H: The mir-34 family in cancer and apoptosis. Cell Death Differ 17: 193-199, 2010.

22. Zhang C, Mo R, Yin B, Zhou L, Liu Y and Fan J: Tumor suppressor microrna-34a inhibits cell proliferation by targeting notch1 in renal cell carcinoma. Oncol Lett 7: 1689-1694, 2014.

23. Aranha MM, Santos DM, Xavier JM, Low WC, Steer CJ, Sola $\mathrm{S}$ and Rodrigues CM: Apoptosis-associated micrornas are modulated in mouse, rat and human neural differentiation. BMC Genomics 11: 514, 2010.

24. Yamakuchi M: Microrna regulation of sirt1. Front Physiol 3: 68, 2012.

25. Nogueiras R, Habegger KM, Chaudhary N, Finan B, Banks AS, Dietrich MO, Horvath TL, Sinclair DA, Pfluger PT and Tschop MH: Sirtuin 1 and sirtuin 3: Physiological modulators of metabolism. Physiol Rev 92: 1479-1514, 2012.
26. Kim D, Nguyen MD, Dobbin MM, Fischer A, Sananbenesi F, Rodgers JT, Delalle I, Baur JA, Sui G, Armour SM, et al. SIRT1 deacetylase protects against neurodegeneration in models for Alzheimer's disease and amyotrophic lateral sclerosis. EMBO J 26: 3169-3179, 2007.

27. Ye J, Liu Z, Wei J, Lu L, Huang Y, Luo L and Xie H: Protective effect of sirtl on toxicity of microglial-derived factors induced by lps to pc12 cells via the p53-caspase-3-dependent apoptotic pathway. Neurosci Lett 553: 72-77, 2013.

28. Liu TX, Howlett NG, Deng M, Langenau DM, Hsu K, Rhodes J, Kanki JP, D'Andrea AD and Look AT: Knockdown of zebrafish fancd 2 causes developmental abnormalities via p53-dependent apoptosis. Dev Cell 5: 903-914, 2003.

29. Potts MB, Vaughn AE, McDonough H, Patterson $\mathrm{C}$ and Deshmukh M: Reduced apaf-1 levels in cardiomyocytes engage strict regulation of apoptosis by endogenous xiap. J Cell Biol 171: 925-930, 2005.

30. Bitterman KJ, Anderson RM, Cohen HY, Latorre-Esteves M and Sinclair DA: Inhibition of silencing and accelerated aging by nicotinamide, a putative negative regulator of yeast sir2 and human sirt1. J Biol Chem 277: 45099-45107, 2002.

31. Hori YS, Kuno A, Hosoda R and Horio Y: Regulation of foxos and $\mathrm{p} 53$ by sirt 1 modulators under oxidative stress. PLoS One 8: e73875, 2013

32. Sun MF, Chang TT, Chang KW, Huang HJ, Chen HY, Tsai FJ, Lin JG and Chen CY: Blocking the dna repair system by traditional chinese medicine? J Biomol Struct Dyn 28: 895-906, 2011.

33. Solomon JM, Pasupuleti R, Xu L, McDonagh T, Curtis R, DiStefano PS and Huber LJ: Inhibition of sirtl catalytic activity increases p53 acetylation but does not alter cell survival following dna damage. Mol Cell Biol 26: 28-38, 2006.

34. Tavernarakis N, Pasparaki A, Tasdemir E, Maiuri MC and Kroemer G: The effects of p53 on whole organism longevity are mediated by autophagy. Autophagy 4: 870-873, 2008.

35. Maiuri MC, Malik SA, Morselli E, Kepp O, Criollo A, Mouchel PL, Carnuccio R and Kroemer G: Stimulation of autophagy by the p53 target gene sestrin2. Cell Cycle 8: 1571-1576, 2009.

36. Bloom GS: Amyloid-beta and tau: the trigger and bullet in alzheimer disease pathogenesis. JAMA Neurol 71: 505-508, 2014.

37. Sun Q, Jia N, Wang W, Jin H, Xu J and Hu H: Activation of sirtl by curcumin blocks the neurotoxicity of amyloid-beta25-35 in rat cortical neurons. Biochem Biophys Res Commun 448: 89-94, 2014. 